Proceedings of the 2009 Winter Simulation Conference

M. D. Rossetti, R. R. Hill, B. Johansson, A. Dunkin, and R. G. Ingalls, eds.

\title{
A POST-DISASTER MOBILITY MODEL FOR DELAY TOLERANT NETWORKING
}

\author{
Md Yusuf S. Uddin \\ Department of Computer Science \\ University of Illinois, Urbana-Champaign \\ 201 N Goodwin Ave. \\ Urbana, IL 61801, USA
}

David M. Nicol

\author{
Coordinated Science Laboratory \\ University of Illinois, Urbana-Champaign \\ 1308 W Main St. \\ Urbana, IL 61801, USA
}

\author{
Tarek F. Abdelzaher \\ Robin H. Kravets \\ Department of Computer Science \\ University of Illinois, Urbana-Champaign \\ 201 N Goodwin Ave. \\ Urbana, IL 61801, USA
}

\begin{abstract}
Delay Tolerant Networking (DTN) considers how to provide communication in contexts where it is unreasonable to assume end-to-end connectivity. Network devices exchange buffered messages when they come into communication range; messages may be buffered and carried physically several times before ultimately being received. Service characteristics of a DTN depend intimately on the underlying movement of devices through physical space; correspondingly, an assessment of DTN technology (e.g. routing protocols, message exchange policies, etc.) depends on that same movement. Existing mobility models provided in simulators lack characteristics one expects in post-disaster communication. We propose a mobility model that includes the impact of the disaster on the transportation network, and that models population and relief vehicle movement. We augment the "Opportunistic Network Environment" (ONE) simulator of DTNs with required extensions and show that characteristics of the DTN are very different using the new model than it is under models that ONE currently provides.
\end{abstract}

\section{INTRODUCTION}

Natural disasters turmoil human activity and disconnect communication services such as phone (landline and cell) and the Internet. As a result of a disaster one sees population shifts into shelters, and movement that is dominated by the response force (e.g., rescue, utility, fire, police, national guard). A well managed recovery operation requires effective communication among these working agents. When significant elements of the communication infrastructure are damaged, needed communication may still be provided through the use of Delay Tolerant Networking (DTN) (DTNRG 2005). In a DTN, significant numbers of the communication devices are not only transponders, but are routers as well. When two such devices first enter communication range with each other, they both execute DTN routing protocols, and as a result may exchange messages that they have buffered in preparation for such a contact. In a disaster scenario one imagines that people with hand-held wireless devices (e.g., cell phone, PDA, even laptops), houses with WiFi routers and automobiles with radios can be connected in an effective way to form a functional communication network, and have DTN-enabled devices carried by moving humans and vehicles buffered, establish connectivity, as a function of whatever movement pattern is induced by their activity. We call this a disaster response network.

A disaster response network is one kind of DTN. Where in traditional wireless networks partitions are treated as an abnormality, they are quite normal in DTN; hence end-to-end 'always on' connectivity from the source to the destination never exists. Networking protocols for DTN are a current and active area of research in applications such as bus networks, vehicular networks, sensor networks, and IPN (Inter-Planetary Networks); disaster response is one of the most motivating and significant instances of DTN.

Significantly, the DTN literature lacks a mobility model attuned to distinct characteristics of disaster scenarios. This is particularly problematic as the service characteristics of a DTN depend greatly on the physical mobility of devices whose movement ultimately connects communication islands. The most commonly used models in DTN simulations are 
Random Waypoint or the more accurate Map-Based Movement Model. But these models do not capture the most salient features of movement at a disaster-population movements are clustered, directed, and transient; during recovery a pattern of movement of among emergency responders dominates. These types of movement are neither completely random nor completely periodic - the defining characteristics of the existing random movement models. To this end, we develop the Post-Disaster Mobility Model (PDM) to model the movement of people and rescue activities in the affected area. The model has been implemented as an extension to the widely used DTN simulator "Opportunistic Network Environment" (ONE) (Keränen and Ott 2007) where we observe significant differences in delay-based connectivity metrics on scenarios where we model mobility using existing models, and PDM. This shows that movement characteristics highlighted by PDM do in fact significantly affect the predicted behavior of the network, and therefore need to be part of the model.

The paper is organized as follows. In $\S 2$ we describe related work, followed by $\S 3$ where we describe in detail the proposed movement model. $\S 4$ explains how the model is implemented using ONE, $\S 5$ presents the results of simulations that highlight the difference in DTN behavior as a function of mobility model. $\S 6$ mentions future extensions and $\S 7$ concludes the paper.

\section{RELATED WORK}

Mobility modeling is an active area of research. The simplest (and most frequently used) model is the Random Walk mobility model. Here an entity moves continuously, as a piecewise linear function of time. Given the end of one linear segment, the next is obtained through a random choice of new direction, and random choice of speed and distance. Velocity along a segment is constant. Another basic model is Random Waypoint (RWP) (Johnson and Maltz 1996) that introduces a random pause time between line segments in the random walk.

Usually the direction angle, speed and pause times of RWP mobility are sampled from a uniform distribution. GaussMarkov model is a variant where the speed and direction value are obtained from the positive Gaussian distribution (where all samples are bounded from below by $\varepsilon>0$ ). Rhee et al. (2007) use the Levy Walks movement model that is very similar to random walk, except that the flight lengths and pause times are drawn from a power law distribution. Although these models produce meeting statistics similar to many real world traces, they do not capture characteristics such as heterogeneity among entities, repetitiveness, group mobility or any relationships between entities.

There are many situations where it is necessary to model the behavior of entities as they move together. For example, a group of people (say, family members) travel together and visit places for common purpose (e.g., shopping). In order to model such situations, a group mobility model is proposed. Here entities are clustered and move as a group. Two most notable group mobility models are Exponential Correlated Random Mobility model (Hong et al. 1999) and Reference Point Group Mobility model (Sanchez and Manzoni 2001, Sanchez 2002).

The community based mobility model (Musolesi and Mascolo 2006) is based on the idea that entities favor squares with higher social attractivity. The social attractivity is based on how many friends are in the same square. Changing friends depending on the time of day results in periodic patterns such as people meeting their work colleagues in the day and their family in the evening. This model lacks group movement and the movement is relatively homogeneous. In the time-variant mobility model (Hsu et al. 2007), entities move to different squares at different times of day in a periodic manner, thereby creating some heterogeneity in both time and space. Entities do not move in groups and the movement is homogeneous in the sense that every entity follows the same instructions.

The most recent mobility model covering aspects such as community, daily routines, heterogeneity, etc. is the Working Day Movement (WDM) model proposed by Ekman et al. (2008). This model presents the everyday life of people who go to work in the morning, spend their day at work, and commute back to their homes at evenings.

In (Nelson, Harris III, and Kravets 2007), authors describe a role-based and event-driven mobility model for disaster recovery networks. The disaster is modeled as events that attract (repel) people with certain roles to (from) the disaster area, as like civilans flee from the disaster area, whereas polices and ambulance frequent to the incident area. The model uses the notion of gravity from physics to compute 'force' applied on individual that reflects in their moving speed when they join to or leave from the scene. The model, an extension on random walks, seemingly simulates the chaos at 'disaster in action' and is most suitable for live hazardous events like 'sudden fire in a building'. In contrast, we model mobility for post-disaster scenario in a very large scale where chaotic random movement is dominated by regulated rescue operation.

\section{POST-DISASTER MOBILITY MODEL}

We develop a new mobility model, the Post-Disaster Mobility (PDM) model, that mimics the situation after a natural disaster. PDM describes different role-based movements, based on a given city map. It models two main groups after a disaster: 
Uddin, Nicol, Abdelzaher and Kravets

survivors, and rescue workers that aid survivors. PDM describes movement models for both groups. Clearly a post-disaster scenario is entirely dependent on the type of disaster. Hurricanes, tornadoes, earthquakes, and fire all induce different movements. One distinguishing characteristic is whether a population is forewarned, such as in a hurricane or tornado, as the population movement may significantly precede the event; with a fire or earthquake the population shifts occur after the event, and may interfere with the emergency response. Recognizing the difference and the fact that we can model the population shift one way or the other, in this paper we concentrate on scenarios where a fore-warned population moves in advance of the disaster to evacuation centers. They stay at these centers for a substantial amount of time and then return to their homes. Following the disaster a relief operation is launched to help survivors (e.g., supply food and water). Our model assumes there are a small number of main coordination centers, and a larger number of evaluation centers. Vehicles move between the main coordination centers and the evacuation centers to supply relief goods. These are some of the vehicles assumed to have DTN devices that buffer and carry communication between communication-isolated coordination and evaluation centers. In addition, a number of rescue workers and volunteers are deployed at each evacuation center to dispense relief goods and services to the affected people (these also serve as DTN "carriers"); other DTN carriers include police officers, who patrol a larger area, e.g. to prevent looting.

Although we try to model mobility in a post-disaster situation, it is hard to directly validate the model against a real disaster instance. No documents/reports happen to describe the disaster operation in as detail as required to reproduce the scene by a simulator. We consulted few formal documents prepared by FEMA (http://www.fema.org) in our construct, at least to the level of identifying various participants and their functionality in the disaster response. National Response Framework NRF (2001) provides guidelines how to mobilize resources and personnel to carry out certain response functions. NRF applies functional approach that groups capacities into 15 Emergency Support Functions (ESFs) to provide resource, personnel, program implementation and emergency services that are likely to be needed during the incident (NRF-Docs 2001). A few ESFs that are being simulated to some extend in our model are transportation (ESF \#1), fire-fighting (ESF \#4), public health and medical services (ESF \#8), urban search and rescue (ESF \#9), and public safety and security (ESF \#13). Our selection of centers and agents is in compliance with this framework.

Incident Command System ICS (2001) is a standardized on-scene incident management concept. It describes how command hierarchy is maintained among various working teams and units. It proposes establishment of ICS facilities, namely incident command post, base or staging area to coordinate rescue operation in the incident area. Base is the location from which the primary logistics functions are coordinated, whereas the staging area hold personnel and equipments waiting for tactical assignments. ICS suggests establishing one or two Emergency Operations Centers (EOCs) which are responsible for community-wide resource management. Another document we consulted is the National Planning Scenarios (Scenario 10: Natural Disaster - Major Hurricane) (Scenes 2001) that describes the components of a response operation after a Category 5 hurricane. It entails the mission objectives such as emergency management/response, hazard mitigation, evacuation/shelters, victim care, recovery/remediation, and so on. In our model, we try to mimic a couple of these response services with the associated agents and their mobility. It is to understand that instead of realizing the exact rescue operation, what we do is to extract the key mobility patterns experienced by the agents and to build our simulator accordingly.

In our mobility model, we address a number of characteristics, described below.

\subsection{Disaster Area and Neighborhoods}

We begin with a map of the disaster area (Figure 1(a)). The map contains connected road segments onto a 2-D plane where possible movements of people and vehicle can occur. We assume that human lives in clustered neighborhoods and a few such neighborhoods are affected by the disaster. To construct neighborhoods, we randomly choose points on the map as neighborhood centers that are far away from one another by certain distance (say, 500m). Then, we place houses randomly around every center within a certain radius (say, 200m) from the center. Once the houses are built, people are created and are randomly assigned to particular houses. The house to which a person is assigned to is treated as his/her home. Figure 1(a) and 1(b) shows the city's neighborhood centers and houses respectively. The various values - the number of total neighborhoods, houses per neighborhoods, total number of people, minimum distance between neighborhoods, the radius of neighborhood - are parameters to the model.

\subsection{Evacuation and Return}

When the evacuation is announced, each human waits a random period of time, then decides whether or not to move. Those that move (chosen randomly, using a "probability of evacuating") go to the nearest evacuation center, where they wait for another random period of time, and then return home. People that do not evacuate, and those that have returned from 


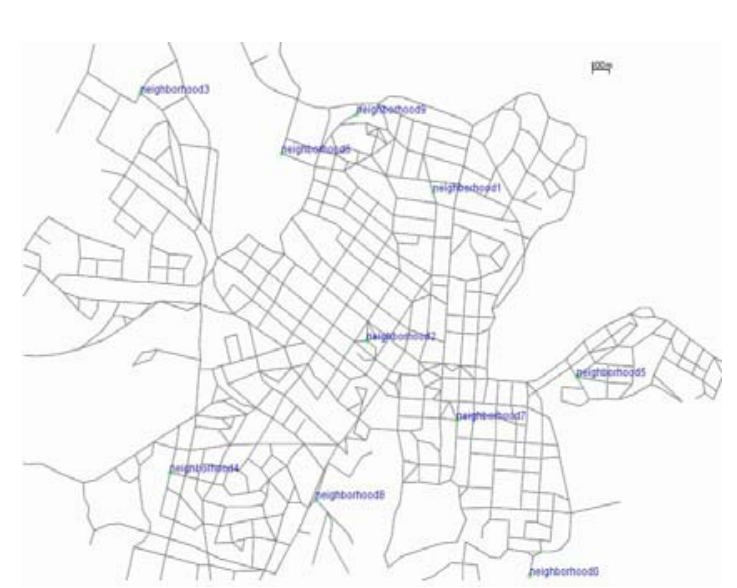

(a) City Map

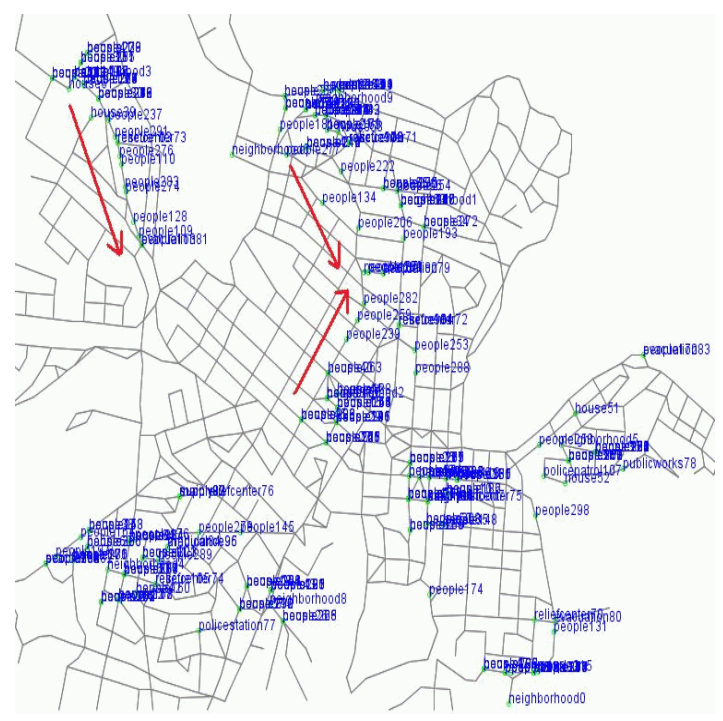

(c) People moving to evacuation centers

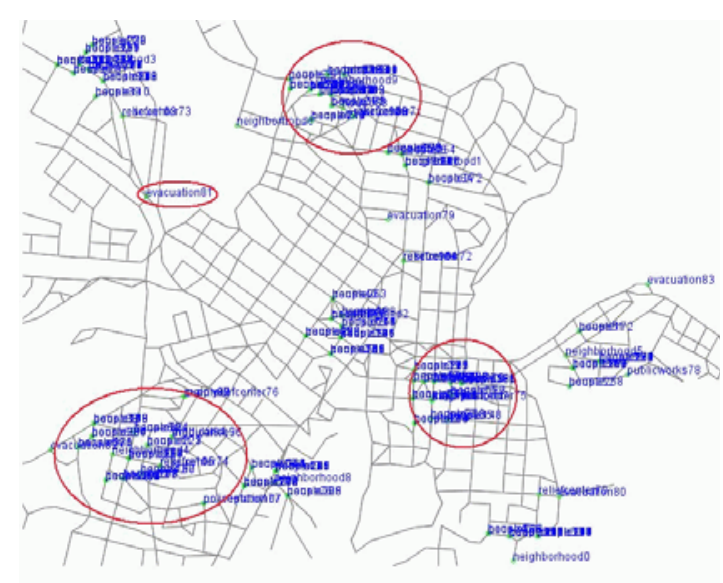

(b) Neighborhood in the city

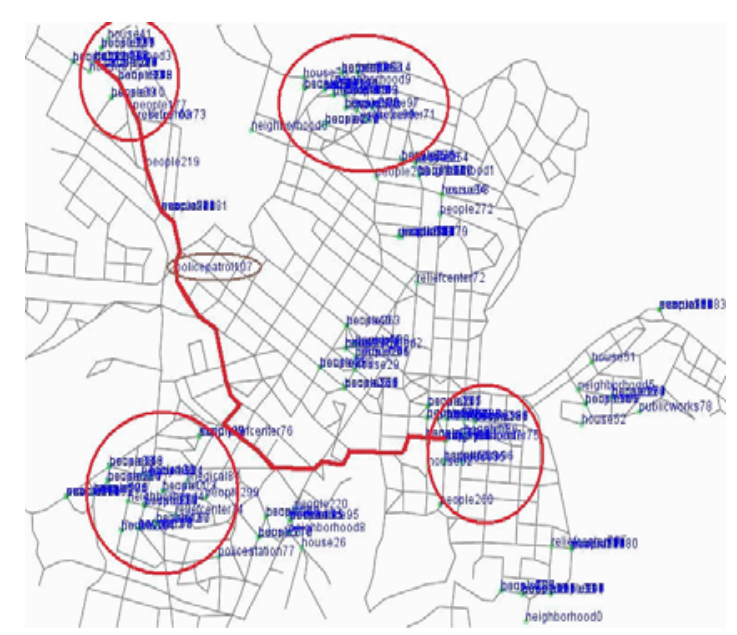

(d) Police patrol

Figure 1: Various components of the mobility model

evacuation randomly move around within their neighborhood. Figure 1(c) pictorially shows that people are moving toward the evacuation centers, as modeled in ONE.

\subsection{Post-Disaster Relief Operation}

The relief operation begins after the disaster. A set of centers are declared to participate in the recovery operation. A few centers are pre-established (e.g., fire-stations), and a few are prepared in some premises for rescue and relief operation (e.g., medial centers, relief camps). There are three key components in modeling the disaster operation - placements of centers, mobile agents, and their interactions.

\subsubsection{Centers}

Our model includes a number of different center types (irrespective of various names - centers, camps, or stations, we refer them all as centers): 
- Main coordination centers: One or two coordination centers (Emergency Operation Centers) are established that coordinate the entire rescue and recovery operation. Rescue teams are formed here and dispersed into different neighborhoods. These centers are also the repositories of all kinds of relief goods and rescue equipments.

- Relief camps: A few relief camps are located. Rescue workers deployed at a neighborhood emanate from these camps, visit houses and return to the center. Relief camps collect their relief material and equipments from the main centers.

- Evacuation centers: At least one evacuation center is established in each neighborhood. These are the places where people are evacuated to when hurricane is warned.

- Medical centers and hospitals: A center that supplies essential paramedics, medical relief, first-aid kits to injured people at evacuation camps or houses. Ambulances at hospitals are also ready to serve emergencies.

- Police and fire station: Police officers regularly visit the evacuation centers and neighborhoods. Police patrols are dispatched from a police station periodically. Fire-trucks are kept at the fire-station for any emergency.

\subsubsection{Mobile Agents and Mobility Patterns}

Rescue workers are the main moving agents in the disaster response, as well as vehicles running between centers, camps, and stations to carry supplies, services, and aid workers. We constrain all movement to take place along the streets on the map, whereas centers can be located in any intersection of streets. We identify four basic types of mobility patterns undertaken by the agents (Figure 2(a)).

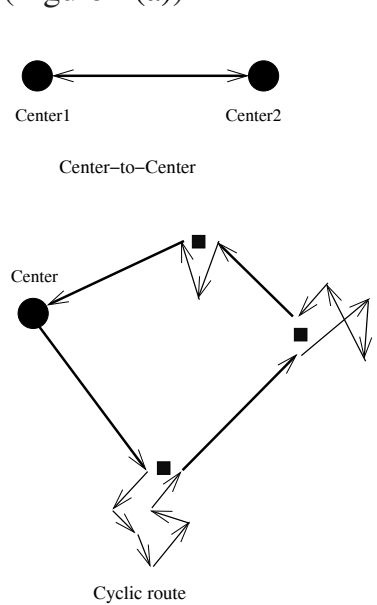

(a) Four different mobility patterns
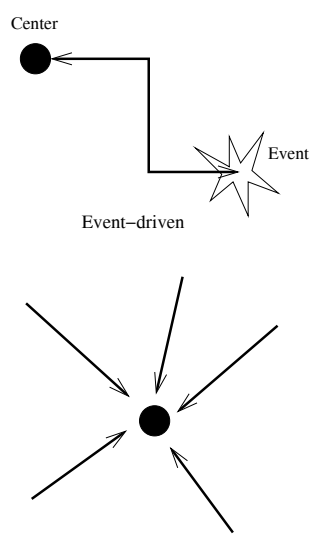

Convergence-Move

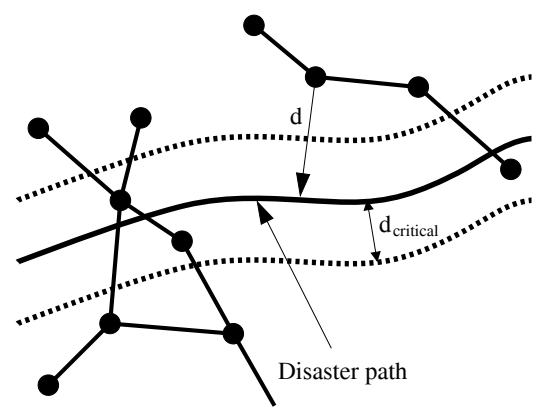

(b) Effect of disaster

Figure 2: Mobility patterns and effect of disaster

- Center-to-Center: This mobility is observed by vehicles which travel back and forth between a set of designated centers, camps or stations. In each trip starting from its home center, the agent picks a destination center from a set, finds a route, and moves toward the destination. After reaching the destination, it waits for a random duration as service time. After the service at the destination, it returns back to the home center. The oscillation repeats during the operation with some pause time in between.

- Event-driven: This type of movement is made when a specific event is notified to a designated center and the associated agent (i.e., vehicle) visits the incident area. After the service, the agent returns to the base. This movement does not however oscillate, but occurs once as event triggers.

- Cyclic route: Some agents take cyclic route from a particular center, visits a few locations of interest and returns to the home center. This is mainly observed by police as a part of their patrol, or any public transport system (bus/tram routes). After visiting each location, agents can optionally take a random walk around the location for a while before heading to the next location.

- Convergence-Move: A set of agents with a particular role/duty get back to their reporting center around a certain fixed time, e.g., when all rescue workers are called back to relief camp for some special instruction. The opposite pattern is the divergence-Move. 
Our model includes the following moving agents:

- Supply vehicles to carry relief goods between main centers and evacuation centers. They follow center-to-center mobility pattern.

- Rescue workers at each neighborhood to help people to evacuate, and later to assist people with relocation. They move from the relief centers to houses and the reverse.

- Ambulances and fire trucks that respond to emergencies (i.e., event-driven mobility). Emergency events are generated at random locations at random times. An ambulance or fire truck starts from its respective home center to visit the target place. After the on-site service (with random duration) the vehicle returns back to the home center.

- Police patrol cars originate from police stations and regularly visit neighborhoods. Obviously, they take the "cyclic route' pattern. At the beginning of a patrol, a set of neighborhoods are chosen randomly. The patrol car starts from the police station and moves to the first neighborhood center. After reaching the neighborhood, it randomly visits a couple of locations (blocks) in that neighborhood with a random wait time at every location. Then, it chooses the next neighborhood and visits a few places there. Finally it returns to the police station. After a while, it picks another patrol. Figure 1(d) shows a police patrol route.

- Volunteers: People within a neighborhood join with rescue workers and move randomly in the neighborhood. The difference between volunteers and the rescue workers is that volunteers do not report to the relief camps, rather they return to their homes.

\subsubsection{Interaction among agents}

Moving agents are equipped with communicating devices that are capable of transferring data when they are within the radio range of each other. There are usually short range radio (say, 50m range) with pretty low data transmission rate. Generally, people (rescue workers, volunteers) keep battery-powered hand held devices (e.g., PDA), while for vehicles these devices are carried by the respective drivers. Each center (or camp/station) is also equipped with similar communicating devices which opportunistically relays information among vehicles or people when they pass by. All these devices are called "DTN routers". While moving around and meeting now and then, they form a disrupted but functional store-carry-and-forward type network that provides communication opportunity to the rescuers and survivors.

\subsection{Effect of Disaster}

The disaster may damage infrastructures and transportation systems, and so limit mobility. We assume a disaster can block road segments and can potentially disconnect communication between the neighborhoods. We develop a simple model to mimic the effect of disaster on the road network as follows.

We assume that the disaster has an intensity $I(1 \leq I \leq 10)$ that reflects the potentiality of its damage. Since we consider hurricane or tornado like disaster, this value may correspond to the category of the hurricane (e.g., category 4$)$. The intensity value 1 represents the weakest disaster whereas the value 10 dictates the strongest. We assume that the track of the storm is a curve that pass over the disaster area with a constant speed. When it passes over the area, all road segments "near" to the path are damaged, with nearness being parametrically specified as follows. Let a point $P$ be $d$ distance away from the disaster path and the factor damage measure the amount of damage incurred at point $P$. We compute damage $(0.0 \leq$ damage $\leq 1.0)$ at $P$ by

$$
\text { damage }=\left\{\begin{aligned}
1.0 & \text { if } d \leq d_{\text {critical }} \\
K \cdot \frac{I}{\left(d-d_{\text {critical }}\right)^{2}} & \text { otherwise }
\end{aligned}\right.
$$

Any point within the critical distance $d_{\text {critical }}$ is completely damaged. Otherwise, the damage is inversely proportional to the square of the distance from the disaster path. The $K$ in Equation 1 is a constant of proportionality that can be computed

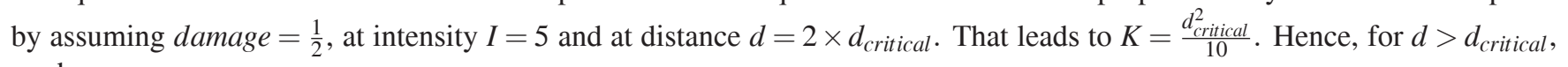
we have

$$
\text { damage }=\min \left(1.0, \frac{d_{\text {critical }}^{2}}{10} \cdot \frac{I}{\left(d-d_{\text {critical }}\right)^{2}}\right)
$$


Uddin, Nicol, Abdelzaher and Kravets

A road segment is unusable if any end of that segment suffers damage more than some threshold (we used 0.5 in our experiments.)

\section{IMPLEMENTATION}

We extended the Opportunistic Network Environment (ONE) simulator (Keränen and Ott 2007) (developed by a different research group than ourselves, and used widely in the DTN community) with our new mobility model. ONE is a highly customizable communication network simulator for delay tolerant networking that has several movement models implemented that import map data and constrain entity movement to the streets and roads of the imported data. ONE can also visualize the imported map and entity movement using a GUI which helps on validating the model in an intuitive way (Ekman et al. 2008). All map-based movement models obtain their configuration data using files formatted with a subset of the Well Known Text format. These files can be edited and generated from real world map data using Geographic Information System software such as ArcView, or OpenJUMP (www.esri.com, www.openjump.org). With map-based movement models, the entities move using roads and walkways from the map data. In addition, different entity groups can be assigned to different maps, constraining their movement in certain parts of the entire map.

ONE comes with three types of map-based movement models. The Map-Based Movement model is a derivative of the Random Walk model, where entities move to randomly determined directions on the map following the roads. Random pauses occur at the waypoints. The Shortest Path Map-Based Movement model is a derivative of the Random Waypoint model, where at decision points entities choose a random destination and then follow the map-based shortest path to that destination. It is also possible in ONE to specify in the configuration file deterministic routes for entities to follow.

\subsection{Extension to ONE}

We added new map based movement models to ONE, extending the Shortest Path Map-Based Movement Model. These are listed in Table 1.

Table 1: Newly added Movement classes to ONE

\begin{tabular}{ll}
\hline Class Name & Purpose \\
\hline StaticMovement & places immobile centers, camps, houses, police stations, on the map \\
BwtnCenterMovement & movement of vehicles between centers; \\
HumanMovment & movement of humans; move to evacuation centers and return; \\
RescueWorkersMovement & $\begin{array}{l}\text { rescue workers; travel between evacuation center and houses } \\
\text { PolicePatrolMovement } \\
\text { movement of police officers patrolling around the area }\end{array}$ \\
\hline
\end{tabular}

These movement models are a cross between completely deterministic specification, and completely random movement. In BwtnCenterMovement a vehicle cycles between two sets of centers. The sets themselves are deterministically specified by the configuration file, but for each travel segment a member of the destination set is chosen randomly. A vehicle may pause for a random duration at a cycle as well. The RescueWorkersMovement model is similar, with a rescue worker cycling between a set of centers and a set of houses in a neighborhood. The PolicePatrolMovement models a patrol car that moves from its home base and visits a random sequence of neighbors, before returning to its home base.

ONE is written in Java. Each movement model is implemented as a class which implements the generic MovementModel interface. The interface MovementModel has two abstract methods that any extended class must override.

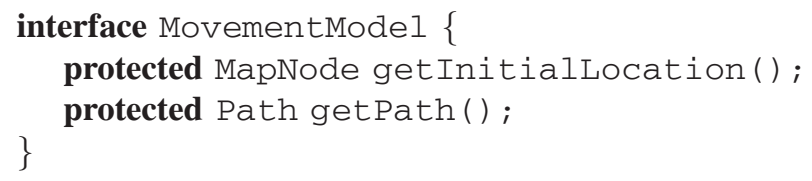

The method getInitialLocation returns the initial location of the entity, while getPath returns the next path (an array of road segments) to the new destination. A particular movement model has to override these two methods depending on what specific computation that model needs to make. 
Uddin, Nicol, Abdelzaher and Kravets

\section{SIMULATION}

We simulated PDM in the ONE system, to compare connectivity characteristics obtained under PDM with those predicted under the existing mobility models in ONE. The principle ones we evaluate are:

- Inter-meeting time: Inter-meeting time is the time interval between contacts for a router pair. It is defined as the time interval during which a pair of routers are out of reach . A contact happens when two routers meet. Actually, meeting is referred to as the event when two routers are able to communicate. Since, all communication is done by radio signal, two routers are in meeting if they are within radio transmission range of each other. We use $50 \mathrm{~m}$ transmission range.

- Neighbor counts : How many other DTN routers does a router tend to see "often"?

- Message delay : How long does it take for a message to be carried from source to destination?

\subsection{Configuration Parameters}

ONE configures itself based on input. To illustrate we show the settings for neighborhoods below. We place 10 neighborhoods (group 1) randomly located on map 1 (several map files can be configured), where neighborhoods would be at least $500 \mathrm{~m}$ away from each other, allowing houses to be located within $200 \mathrm{~m}$ of neighborhood center. Neighborhoods are immobile, so they use StaticMovement model (which is already part of ONE). We also show settings for supply vehicles (Group 5). They move between main coordination centers and evacuation centers. A vehicle's speed is sampled from a positive normal with mean $50 \mathrm{~km} / \mathrm{hr}$ and standard deviation $5 \mathrm{~km} / \mathrm{hr}$, while wait time is distributed uniformly between 20 mins and 60 mins.

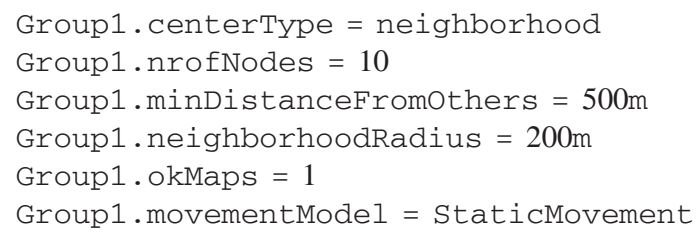

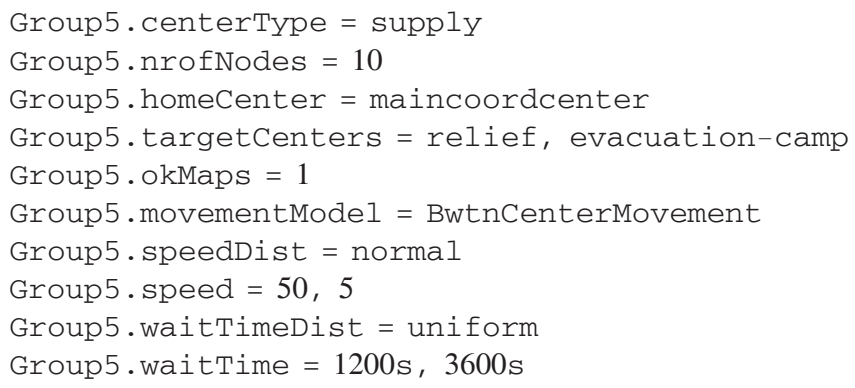

Here we see use of one of our new mobility patterns BwtnCenterMovement that describes movement between centers. We also use StaticMovement for designated centers.

We simulate for 96 hours for a disaster with intensity 5, in a model with the characteristics shown in Table 2 .

Table 2: Model settings

\begin{tabular}{llll}
\hline Type & Number & Type & Number \\
\hline Neighborhoods & 10 & Coord. to Relief supply vehicles & 5 \\
Houses & 60 & Relief supply to Evacuation center vehicles & 5 \\
Coordination center & 2 & Ambulance & 2 \\
Relief supply center & 10 & Road repair vehicle & 1 \\
Police Station & 1 & Police patrol & 1 \\
Evacuation center & 5 & Rescue workers & 20 \\
Medical center & 1 & People & 200 \\
\hline
\end{tabular}

In this simulation we assumed that every driver of a vehicle carries a communication device that also serves as a DTN router, and that every center has a DTN router. We assume that all communication occurs by DTN-routing between routers that come in close-enough proximity (within $50 \mathrm{~m}$ ), and that every modeled person carries a DTN router. Most of the connectivity is supplied by the movement of disaster response vehicles between various centers. The map used is the one supplied with ONE (a portion of Helsinki). 
Uddin, Nicol, Abdelzaher and Kravets

\subsection{Results}

For the purposes of Delay Tolerant Networking, the time between when DTN routers meet is an important determinant of how quickly physically carried messages pass between carriers. A key distinctive of PDM over the other models available in ONE is PDM's ability to focus on targeted sets of destinations. To see the impact this has on inter-meeting time, we partition the set of routers determined by the type of their home center (i.e., supply, rescue, or police). Each measured inter-meeting time is recorded in the set(s) of measurements corresponding to the partition of the routers who meet. We then compute an empirical complementary cumulative distribution function over all observations gathered in five independent simulations of 96 hour scenarios. This is illustrated in Figure 3, for the PDM model, and the map-based Random MapPoint model (RMP). RMP is the map-variant of random waypoint movement (RWP). Vehicle speeds are constant on all road segments, eliminating speed from having an influence on the comparison. For each router we measure the time between its successive meetings with other routers, and aggregate the measurements for routers whose home base centers are the same. The plot lists the times for routers from emergency supply centers, police centers, and evacuation centers. The difference in scale of the distributions is striking between PDM and RMP; PDM is a factor of two or three faster in each category. The differences are summarized in Table 3 in terms of the mean and standard deviations of inter-meeting times.

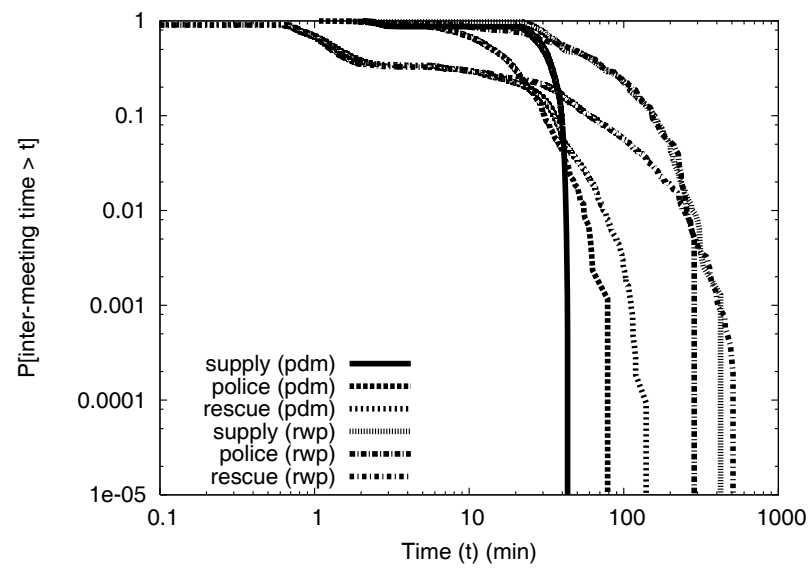

Figure 3: Complementary CDF of Inter-meeting time, based on type of home base of DTN router

Table 3: Mean inter-meeting times for different vehicles

\begin{tabular}{|l|cc|cc|}
\hline Center type & \multicolumn{2}{|c|}{ RMP } & \multicolumn{2}{c|}{ PDM } \\
& mean & stdev. & mean & stdev \\
\hline Supply & 70.12 & 7.01 & 27.99 & 0.82 \\
Police & 67.54 & 14.94 & 16.60 & 1.28 \\
Rescue & 20.98 & 2.20 & 10.6 & 0.54 \\
\hline
\end{tabular}

Recall our definition that two routers are neighbors if they meet at least 10 times during the scenario run. Figure 4(a) plots the number of neighbors for each router in the network. Since PDM allows repetitive movement where a few routers visit the same places repeatedly, we expect there to be a larger neighborhood among routers than under models with less structure in their movement. We see that most routers have a small number of neighbors (average 15). Yet, some routers (those with Id 85 - 107) have very many neighbors. We zoom this part to illustrate clearly in Figure 4(b). These correspond to supply vehicles, rescue workers, ambulances, and police patrol; this is very intuitive, given their wide ranging mobility.

By way of comparison, Figure 4 shows the neighbor count for Random MapPoint mobility, assuming meeting radii 50 and $10 \mathrm{~m}$ (i.e., the proximity needed for connectivity). Here the neighbor with radius $50 \mathrm{~m}$ is moderately high, but drops to nearly 0 with the smaller radius. Contrast this with neighbor counts of PDM at 10m, which are essentially the same as they are at $50 \mathrm{~m}$. This occurs because moving routers pause at centers and meet other routers in close vicinity.

End-to-end message delivery time is a very direct evaluation of how the mobility model affects DTN behavior. Under PDM mobility one expects that message exchanges happen frequently at centers, as these are directed destinations for many of the movements. Traffic generation defines a Poisson stream (with rate 0.5 messages per hour) between each ordered pair of centers. The message generation phase endures for 48 hours of the 96 hour simulated scenario. Under RMP mobility 


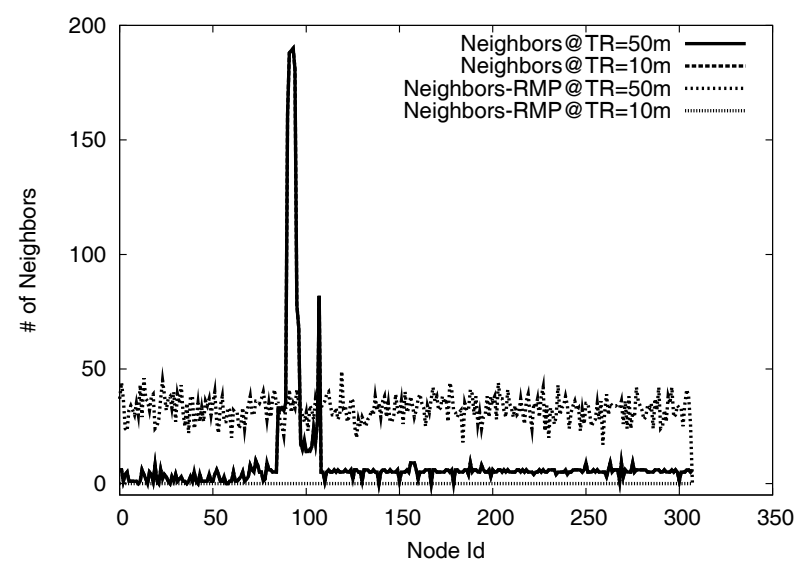

(a) Neighbor counts

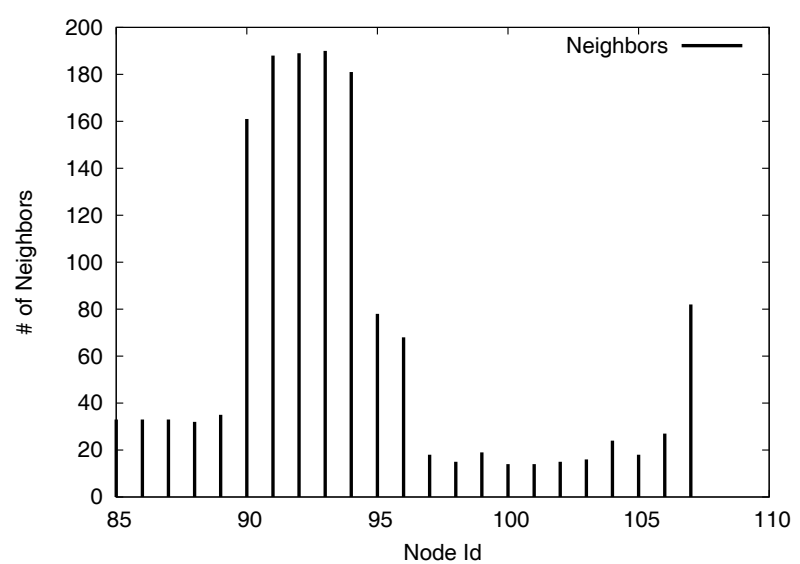

(b) Higher neighbor counts

Figure 4: Neighbor counts

a message generated at a source center is picked up by the next vehicle that randomly happens to pass the center; other message exchanges are likewise due to random drive-bys of vehicles, until ultimately a vehicle randomly happens to pass the message's destination center.

Figure 5(a) plots the mean and standard deviation of a message's delivery time, as a function of the class of its source and destination. Messages are routed by the DTN routing protocol MaxProp (Burgess et al. 2006). MaxProp is a flooding based scheme with a prioritization of messages in buffer. We work on three center classes: main coordination centers (main), evacuation camps (ea) and relief centers (rlf). The measurements are derived from five independent simulation runs, where the measurements from all runs are aggregated.

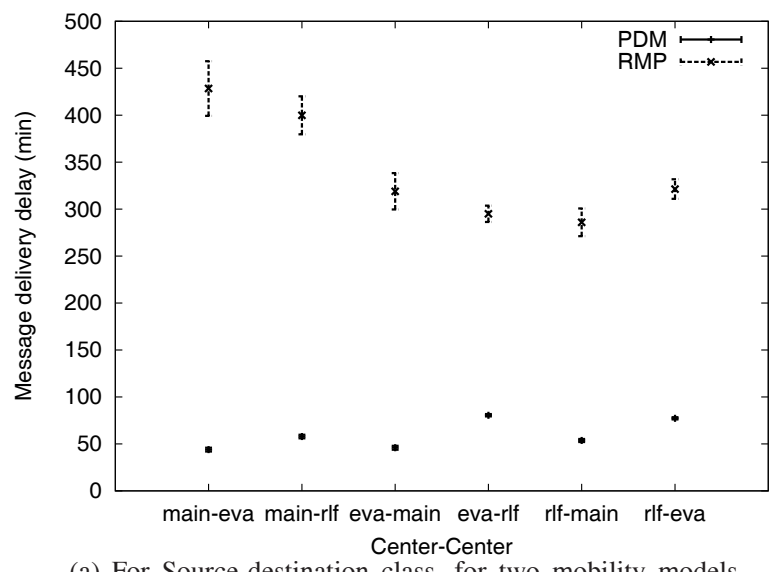

(a) For Source-destination class, for two mobility models

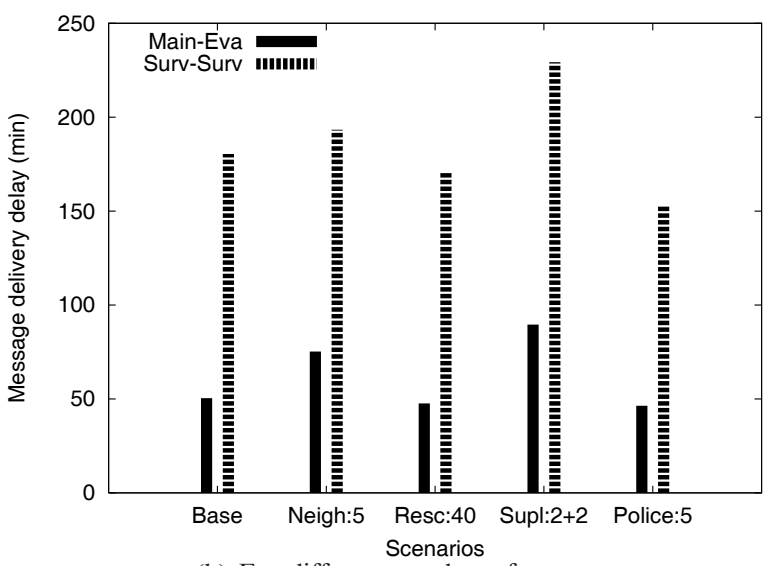

(b) For different number of agents

Figure 5: Message delivery times as a function of source-destination pair type and number of agents of a particular type

The most striking features of this data are that PDM is again significantly faster in message delivery-four to ten times faster-and that in both models there is a surprising lack of variability, when viewed in terms of source-destination classes. Had we presented the statistic's variability taken over all classes we would see structural variability due to map distances, inter-mixed with variability due to the mobility model itself. This figure shows that, at least for this set of experiments, the structural variability dominates.

Figure 5(b) demonstrates the message delay for two kinds of traffic - main center to evacuation camps and survivors to survivors - when the number of a particular agent is changed from the base setting (Table 2). Survivors reside at neighborhood houses or in evacuation camps and pass messages to another survivor in the same neighborhood or another. We see that message delivery among the survivors take more time than main centers to evacuation camps. This is because messages need to pass more hops (and wait more thereby) to reach distant neighborhoods. We also observe that number of agents 
has significant impact on delay. For instance, decreasing the number of supply vehicles substantially increases the delay, whereas deploying more police patrols reduces it.

\section{COMING EXTENSIONS}

There are a number of features that will enhance PDM's ability to capture post-disaster dynamics. At present the model concentrates on relief activities and vehicle movements among centers. The models of population movement are very simple, and (critically) the speed of people and vehicles on road segments are deterministic or random. A striking characteristic of population movement in the midst of a hurricane evacuation is road congestion, which affects both the population and the response personal. Our future work will correct this deficiency. Another useful feature will be a broader set of models for capturing the effects of a disaster on the transportation and communication infrastructure. A hurricane model would focus on damage due to flooding; a fire model would make the disaster effects start locally, and grow dynamically. Great potential exists for integrating networking simulation like ONE with standard and more sophisticated GIS databases, and infrastructure descriptions.

\section{CONCLUSION}

Delay tolerant networking is an emerging area of study for providing connectivity in scenarios where end-to-end connectivity is not normal. The fact that communication infrastructure is often crippled in the wake of natural disasters suggests post-disaster response as a compelling application domain for DTN techniques. However, design of hardware and software to best create a post-disaster DTN requires a realistic assessment of connectivity within the DTN after the disaster. As DTN networks depend heavily on physical movement of devices that can buffer and exchange messages with other DTN devices, the behavior of a DTN would seem to depend on the underlying mobility assumed of the vehicles and people carrying DTN-enable devices. Recognizing this, we have developed a movement model for post-disaster scenarios, and extended the capabilities of the most widely used DTN simulator (ONE) to include these movements. To highlight the dependency of DTN behavior on the underlying mobility model, we measured inter-meeting times, and end-to-end message delivery times on ONE simulator which differ only in the assumed DTN router movements. Comparing our model with the most natural existing candidate in ONE, we find that indeed network behavior very much depends on the mobility model. Our work takes the first step in making ONE useful for application of DTN studies in post-disaster scenarios, and future work will augment that reported here to better model population movement during disasters.

\section{ACKNOWLEDGMENTS}

This work was funded in part by NSF grants CNS 06-26825, CNS 06-26342 and CNS 05-54759.

\section{REFERENCES}

Burgess, J., B. Gallagher, D. Jensen, and B. N. Levine. 2006. MaxProp: Routing for vehicle-based disruption-tolerant networks. In Proceedings of the IEEE INFOCOM 2006. Barcelona, Spain.

DTNRG 2005. Delay-tolernat network. <www. dtnrg. org> [Accessed August 13, 2009].

Ekman, F., A. Keränen, J. Karvo, and J. Ott. 2008. Working day movement model. In Proceedings of the 1st ACM SIGMOBILE workshop on Mobility models, MobilityModels '08, 33-40. Hong Kong, China.

Hong, X., M. Gerla, G. Pei, and C. Chiang. 1999. A group mobility model for ad hoc wireless networks. In Proceedings of the ACM International Workshop on Modeling and Simulation of Wireless and Mobile Systems (MSWiM). Seattle, Washington, USA.

Hsu, W. J., T. Spyropoulos, K. Psounis, and A. Helmy. 2007. Modeling time-variant user mobility in wireless mobile networks. In Proceedings of the IEEE INFOCOM 2007, 758 - 766. Alaska, USA.

ICS 2001. National incident management system. Available via <http://www.training. fema.gov> [Accessed August 13, 2009].

Johnson, D., and D. Maltz. 1996. Dynamic source routing in ad hoc wireless networks. Mobile Computing:153 - 181.

Keränen, A., and J. Ott. 2007. Increasing reality for DTN protocol simulations. Technical report, Helsinki University of Technology. <http://www.netlab.tkk.fi/tutkimus/dtn/theone/> [Accessed August 13, 2009]. 
Uddin, Nicol, Abdelzaher and Kravets

Musolesi, M., and C. Mascolo. 2006. A community based mobility model for ad hoc network research. In Proceedings of the 2nd ACM/SIGMOBILE International Workshop on Multi-hop Ad Hoc Networks: from theory to reality (REALMAN'06). Florence, Italy.

Nelson, S., A. Harris III, and R. Kravets. 2007. Event-driven, role-based mobility in disaster recovery networks. In Proceedings of the MobiCom workshop CHANTS 2007, 27-34. Montreal, Quebec, Canada.

NRF 2001. National Response Framework. Available via <http://www. fema.gov/emergency/nrf/> [Accessed August 13, 2009].

NRF-Docs 2001. NRF documents. Available via <http: / /www. fema.gov/pdf/emergency/nrf/nrf-core.pdf> [Accessed August 13, 2009].

Rhee, I., M. Shin, S. Hong, K. Lee, and S. Chong. 2007. On the levy-walk nature of human mobility: Do humans walk like monkeys? Technical report, North Carolina State University, North Carolina, USA.

Sanchez, M. 2002. Mobility models. Available via <http: / /www. disca. upv. es/mi san/mobmodel. htm> [Accessed August 13, 2009].

Sanchez, M., and P. Manzoni. 2001. Anejos: A java based simulator for ad-hoc networks. Future Generation Computer Systems 17:573-583.

Scenes 2001. National Planning Scenarios. Available via

<http: / / www. oregon.gov/OMD/OEM/docs / dom_prep/ fed_support/national_planning_scenarios.pdf> [Accessed August 13, 2009].

\section{AUTHOR BIOGRAPHIES}

MD YUSUF S. UDDIN is a second year Ph.D. student of Computer Science at the University of Illinois, Urbana-Champaign. He obtained his B.Sc. (2004) and M.Sc. (2006) in Computer Science and Engineering from Bangladesh University of Engineering and Technology (BUET), Dhaka, Bangladesh. His research interests include distributed systems, ad hoc networks, and sensor networks. Currently he is working on delay-tolerant networking issues for post-disaster scenarios. His email address is <mduddin2@illinois.edu>.

DAVID M. NICOL is Professor of Electrical and Computer Engineering at the University of Illinois, Urbana- Champaign, and member of the Coordinated Sciences Laboratory. He is co-author of the textbook Discrete-Event Systems Simulation, and served as Editor-in-Chief at ACM TOMACS from 1997-2003. He was the General Chair of the 2004 Conference on Principles of Advanced and Distributed Simulation, and the General Chair of the 2006 Winter Simulation Conference. From 1996-2003 he was Professor of Computer Science at Dartmouth College, where he served as department chair, and at the Institute for Security Technology Studies served as Associate Director for Research and Development, and finally as Acting Director. From 1987- 1996 he was on the faculty of the Computer Science department at the College of William and Mary; 1985-1987 he was a staff scientist at the Institute for Computer Applications in Science and Engineering. He has a B.A. in mathematics from Carleton College (1979), an M.S. (1983) and Ph.D. (1985) in computer science from the University of Virginia. His research interests are in high performance computing, performance analysis, simulation and modeling, and network security. He is a Fellow of the IEEE and a Fellow of the ACM. His email address is <dmnicol@illinois. edu>.

TAREK F. ABDELZAHER is an Associate Professor in the Computer Science department of the University of Illinois at Urbana-Champaign. His research interests include real-time computing, distributed systems, sensor networks, and control. He received his Ph.D. in adaptive real-time systems from the University of Michigan. He is the editor in chief of the Journal of Real-Time Systems and an associate editor of IEEE Transactions on Mobile Computing, IEEE Transactions on Parallel and Distributed Systems, ACM Transactions on Sensor Networks, and Ad Hoc Networks. He's a member of the IEEE and ACM. His email address is <zaher@illinois. edu>.

ROBIN H. KRAVETS is currently an Associate Professor at the Computer Science Department at the University of Illinois, Urbana-Champaign. Dr. Kravets received her Ph.D. from the College of Computing, Georgia Institute of Technology in 1999. She is the head of the Mobius group at UIUC, which researches communication issues in mobile and ad hoc networking, including power management, connectivity management, transport protocols, admission control, location management, routing and security. Her research has been funded by various sources, including the NSF and HP Labs. She is currently a member of the Editorial Board for IEEE Transactions on Mobile Computing and Elsevier Ad Hoc Networks Journal and was an Associate Editor of MC2R: Mobile Computing and Communications Review. For more detailed information, please visit: http://www-sal.cs.uiuc.edu/〜rhk/. Her email address is <rhk@illinois.edu>. 